\title{
A Study on Cognitive Affordance Analysis and BX Design of Flight Reservation Application UI
}

\author{
Joon-woo Park ${ }^{1}$, Kyung-il Doo * \\ ${ }^{1}$ Professor, Dept. of Broadcasting Sound \& Visual, Dealim University, Anyang-si, 13916 Korea \\ *2 Professor, Dept. of Digital Contents Creation, Semyung University, Jechon-si, 27136 Korea \\ jwpark@daelim.ac.kr ${ }^{1}$, artistdoo@semyung.ac.kr*2
}

\begin{abstract}
Article History:Received:11 november 2020; Accepted: 27 December 2020; Published online: 05 April 2021
Abstract: This study checked whether the application of the affordance design, which may improve the usability of the flight reservation application user, affects the brand's value, reliability, and loyalty, and sought an effective UI design plan of the flight reservation application. Researchers also checked the trend of changes in the flight reservation environment through theoretical study, and derived factors to analyze the impact of affordance design on user experience and brand experience. As for main analysis, the effect of the affordance design factor on the user experience and the effect of the usability factor on the brand experience has been checked using statistical analysis, and the effect priority of the affordance design factor by usability factor and the effect priority of the usability factor by brand experience factor have been derived. In the flight reservation application design, the application of the affordance design was identified to have a positive effect on the user experience. In terms of ease of learning, efficiency and memorization, the learning form and the use of metaphor were ranked high, and in the case of error prevention, contextual continuity and use correlation were analyzed as important factors. In satisfaction, all affordance factors were analyzed to form a relatively high satisfaction. The usability factors of the affordance design showed a high degree of effect on the brand's value, reliability and loyalty, and in particular, satisfaction and error prevention were identified as the most direct factor influencing the brand experience.
\end{abstract}

Keywords: Flight Reservation System, Mobile Application, User Interface, Affordance Design, User Experience, Brand Experience

\section{Introduction}

With the popularization of smartphones, most people use mobile application content, and direct reservation using mobile applications increases for flight reservation. This shows that the frequency of flight reservations increases in line with the cultural trend of mobile first. In addition, it shows that the possibility that the various user experiences felt while using the mobile application will directly affect the formation of the image of the corresponding brand increases. In other words, the UI design of the flight reservation application can also influence the formation of the airline's image, and the aesthetics of the design and the positive user experience become an important tool to recognize the brand. Therefore, this study analyzes the effect of the usability of the cognitive affordance design where the user experience of the mobile flight reservation application naturally induces the behavior and the user experience of the flight reservation environment on the brand experience. The researcher intends to find an effective affordance-based UI design plan by deriving the effect on the value cognition, trust and loyalty of the brand.

\section{Theoretical considerations}

\subsection{Flight reservation environment}

Now, we are living in the mobile first era. That is, we live in various technologies and contents due to the increase in the spread of smart devices, and contents optimized for mobile environments such as mobile applications already occupy a large part of our lives

Accordingly, many people reserve the flight directly through airlines or agencies using a mobile application as shown in Figure 1. According to the '2018 National Travel Survey Report', internet sites/mobile apps account for $19.3 \%$ of the total as the path to acquire domestic travel information, and for overseas trips, the same is acquired through internet sites/mobile apps at a high rate of $44.9 \%[1]$. The flight reservation can also be seen as a way to acquire travel information and fall under this statistical category.

*Corresponding author: Kyung-il Doo 

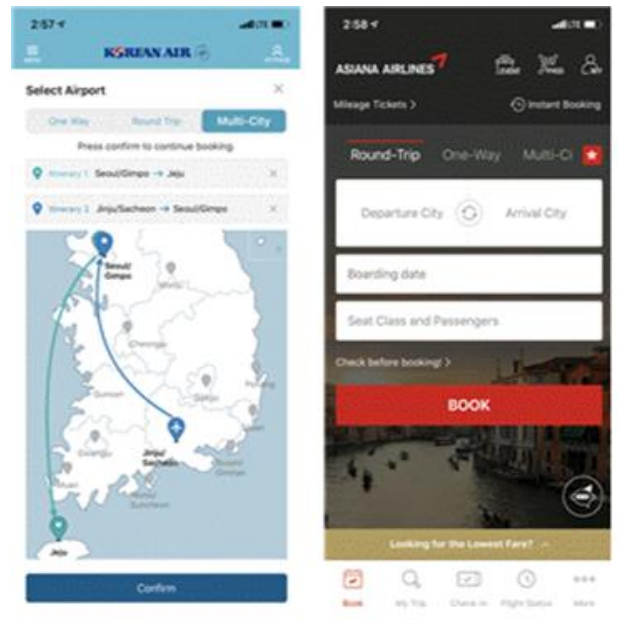

Figure 1. Flight reservation system applications

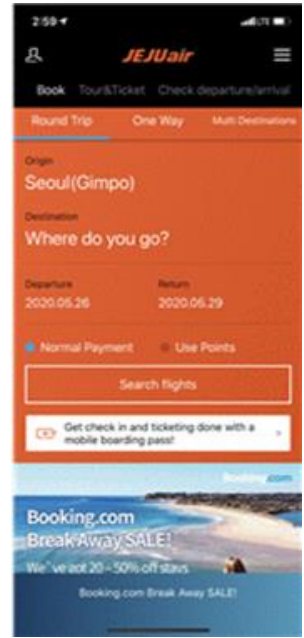

In this way, the mobile application use environment which has been established according to the mobile first trend does not simply seek to increase the convenience of users using smart devices, but now the influence of the user experience on the marketing depending on the degree of convenience, aesthetics and interest of mobile applications increases[2]. In other words, the image of the brand and the company providing the application can be mind-positioned according to the user experience of the mobile application. Accordingly, analysis and improvement work on the flight reservation application UI will be an essential method to reinforce the brand image in this mobile first era.

\subsection{Affordance design}

The term of affordance appeared first time in the book, "The Ecological Approach to Visual Perception," by James Jerome Gibson, an American eco-psychologist. He defined affordance as the relationship between the world and the actor who perceives the environment and take action to form relation with environment. Donald A. Norman, in his book, 'Design and Human Psychology', began to use affordances from the viewpoint of interaction between human and computer.

Classification by factor for research on affordance design of smart device mobile application has been analyzed based on the cognitive affordance. In other words, in A Study on Affordance Design to Induce User`s Cognitive Experiences', the process of applying the factors constituting the UI of mobile application to the cognitive affordance was analyzed in terms of the image in visual dimension, text, metaphor, visual cue of volume and semantic dimension, directivity, current status indication and action expression[3]. In this study, the affordance design of flight reservation application UI design in visual and semantic dimensions is classified as Table 1 below.

Table 1: Classification of affordance design elements

\begin{tabular}{|c|c|c|}
\hline dimension & element & contents \\
\hline \multirow{5}{*}{$\begin{array}{c}\text { visual } \\
\text { dimension }\end{array}$} & image & Visual expressions such as photos and illustrations \\
\hline & metaphor utilization & $\begin{array}{l}\text { Design elements that are more intuitive than text through } \\
\text { metaphorical expressions such as icons }\end{array}$ \\
\hline & learned form & User-learned forms such as shadows and arrows \\
\hline & color & Creating an atmosphere through perceived colors \\
\hline & layout & Composition placed within the frame \\
\hline \multirow{2}{*}{$\begin{array}{l}\text { semantic } \\
\text { dimension }\end{array}$} & contextual continuity & $\begin{array}{l}\text { Contextual elements of achieving goals through continuous use } \\
\text { of content, such as inducing the following actions in the } \\
\text { reservation process }\end{array}$ \\
\hline & use relationship & $\begin{array}{l}\text { Usage relationship of the current content, such as the } \\
\text { completion button is activated only after inputting an article }\end{array}$ \\
\hline
\end{tabular}

\subsection{UX and BX}

Usability evaluates how easy and convenient the user interface can use functions. The word "usability" is also cited in the act to develop "ease of use" in the design process. In addition, usability is said to be a collection of tools that facilitate the user's task achievement. Usability is a factor that forms user experience, and usability factor can be classified and defined as ease of learning, efficiency, memory possibility, error prevention and satisfaction as Table 2 below[4]. 
Table 2: Usability factors and definitions

\begin{tabular}{|c|l|}
\hline usability factor & \multicolumn{1}{|c|}{ contents } \\
\hline learnability & $\begin{array}{l}\text { How easy is it for users to accomplish basic tasks the first time they encounter the } \\
\text { design? }\end{array}$ \\
\hline efficiency & Once users have learned the design, how quickly can they perform tasks? \\
\hline memorability & $\begin{array}{l}\text { When users return to the design after a period of not using it, how easily can they } \\
\text { reestablish proficiency? }\end{array}$ \\
\hline errors & $\begin{array}{l}\text { How many errors do users make, how severe are these errors, and how easily can } \\
\text { they recover from the errors? }\end{array}$ \\
\hline satisfaction & How pleasant is it to use the design? \\
\hline
\end{tabular}

Brand experience refers to the experience that occurs in the relationship between the consumer and the brand and refers to the experience that occurs in combination with the consumer's subjective and intrinsic reactions related to the brand. Brand experience is more influenced by brand-related stimuli. This is closely related to brand identity factors such as name, logo, signage, package, etc., and the brand experience was considered to occur as stimuli related to such brand and consumers' subjective and intrinsic reactions are combined together[5]. From this point of view, Gray Holland presented the "cycle of experience" as shown in Figure 2[6].

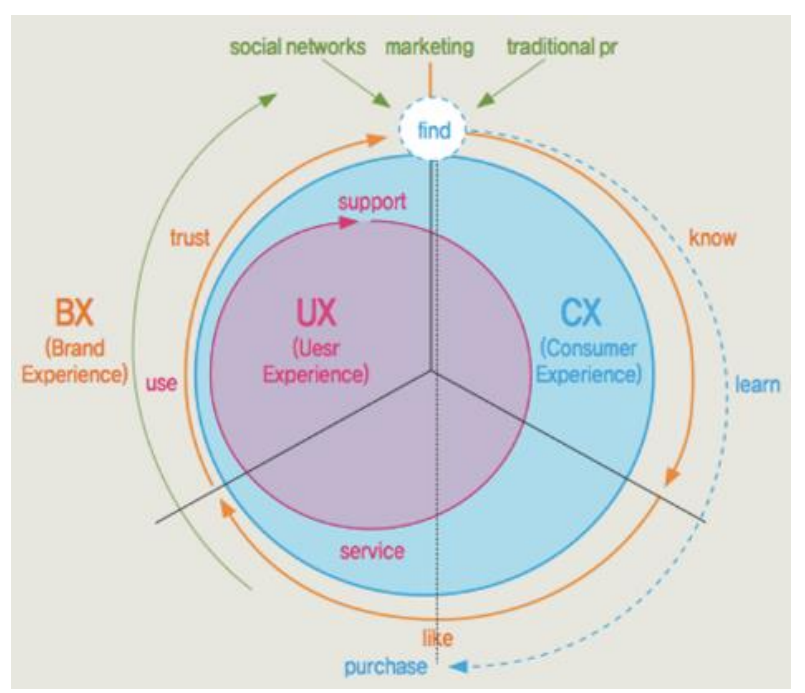

Figure 2. Gray Holland's "cycle of experience"

UX (User Experience), which is a user experience, generated the concept of CX (Customer Experience), a customer experience grafted with a user called a customer in the marketing and public relations sector, and the customer experience came to include BX (Brand Experience), a brand experience according to service preference, reliability, and usability[7,8].

Brand experience occurs when the stimulus related to the brand and the subjective and intrinsic reaction of the consumer are combined and is defined as 'Process and Results of Experience' that are generated by a specific stimulus related to the brand[9]. Accordingly, factors that measure brand experience through usability factors include brand value, reliability, and loyalty as shown in Table 3[10] below, and the brand experience can be measured through these factors.

Table 3: Brand experience metrics

\begin{tabular}{|c|c|}
\hline brand element & contents \\
\hline value & $\begin{array}{l}\text { This brand offers good quality for its price. } \\
\text { - This brand is highly valued overall. } \\
\text { - This brand is better than I thought. }\end{array}$ \\
\hline reliability & $\begin{array}{l}\text { This brand is reliable. } \\
\text { - I have the belief that this brand will provide what I want. } \\
\text { These brands are immersed in fulfilling their promises. }\end{array}$ \\
\hline loyalty & $\begin{array}{l}\text { I want to continue to use this brand. } \\
\text { I think the quality of this brand is excellent. }\end{array}$ \\
\hline
\end{tabular}




\section{BX Analysis of Affordance Design}

This chapter aims to derive the meaning of brand creation experience in flight reservation application that applied affordance design by analyzing the connection between the usability as a user experience according to the affordance design, and the sub factor that creates the brand experience

\subsection{Analysis Method and Process}

The analysis method and process of this study, as shown in Figure 3 below, would like to check the impact of 4 factors of affordance design on 5 sub-factors of the user experience and measure the impact of 5 factors of the usability on sub factors that consist the brand experience.

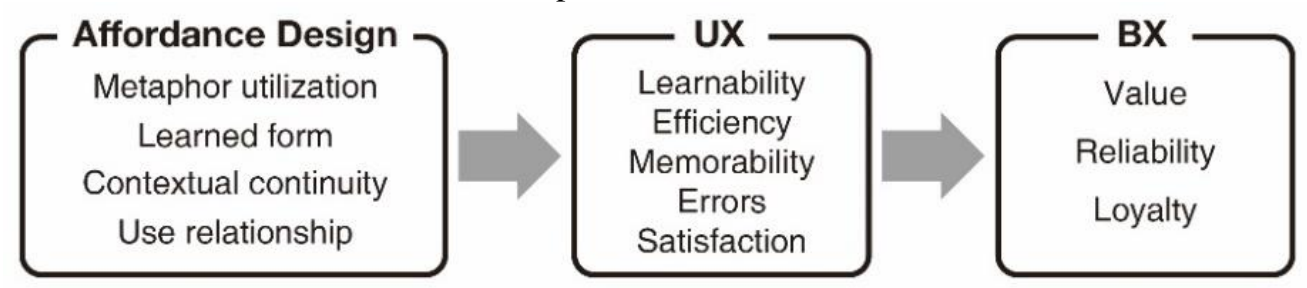

Figure 3. Research analysis model

\subsection{Weight evaluation of Affordance design}

An expert council was formed to select analysis factors among the factors applied to the affordance design of the flight reservation application UI and focus group interviews and weight evaluation were conducted. The expert council consisted of 7 members, including 4 incumbent professors and 3 content UI production experts. The affordance design factors derived through the expert council and focus group interview and weight evaluation are shown in Table 4. Weight evaluation was conducted on 7 factors including image in visual dimension, utilization of metaphor, learned form, color, contextual continuation of layout and semantic dimension, and use relation.

Metaphor that received high weight evaluation, learned form, contextual continuity and use relationships were selected as factors to evaluate the affordance design.

The design factors of metaphor utilization, learned form, contextual continuity, and affordance of use relations received high weight in consideration of the opinion that they play a high role as design factors inducing user behavior in the application UI environment.

On the other hand, in the case of image, color, and layout, they were considered to act as an affordance design factor in the flight reservation application UI environment but were excluded in consideration of the opinion that they are design factors that play the same role in the design of other media.

Table 4: Weight evaluation for selecting affordance design elements

\begin{tabular}{|c|l|c|c|}
\hline element & \multicolumn{1}{|c|}{ reason for selection } & selection & weight \\
\hline image & \multicolumn{1}{|c|}{-} & $\mathrm{X}$ & $2 \%$ \\
\hline metaphor utilization & $\begin{array}{l}\text { Intuitive information delivery through metaphorical } \\
\text { expression }\end{array}$ & $\mathrm{O}$ & $23 \%$ \\
\hline learned form & Improved usability of UI design by utilizing the learned form & $\mathrm{O}$ & $16 \%$ \\
\hline contextual continuity & $\begin{array}{l}\text { It is possible to induce the next action in a sequential line of } \\
\text { action }\end{array}$ & $\mathrm{O}$ & $29 \%$ \\
\hline use relationship & Minimizes user error by clarifying the status of the function & $\mathrm{O}$ & $25 \%$ \\
\hline color & - & $\mathrm{X}$ & $4 \%$ \\
\hline layout & -1 & $\mathrm{X}$ & $1 \%$ \\
\hline \multicolumn{1}{|c|}{ total } & 4 & $100 \%$ \\
\hline
\end{tabular}

\subsection{BX effect analysis}

\subsubsection{BX analysis experiment subjects and method}

For brand experience analysis, in this study, the effect of factors selecting an affordance design through a questionnaire on the user experience and the effect of usability factors on the brand experience were measured. Subjects of the survey were those with experience in flight reservation applications, and the demographic characteristics of the survey subjects are shown in Table 6 below.

Table 5: Demographic characteristics of the survey subjects

\begin{tabular}{|c|c|c|}
\hline \multicolumn{2}{|c|}{ division } & number of responses \\
\hline \multirow{2}{*}{ gender } & male & $36(52.9 \%)$ \\
\cline { 2 - 3 } & female & $32(47.1 \%)$ \\
\hline age & teenagers & $6(8.8 \%)$ \\
\hline
\end{tabular}




\begin{tabular}{|c|c|c|}
\hline \multirow{2}{*}{} & $20 \mathrm{~s}$ & $23(33.8 \%)$ \\
\cline { 2 - 3 } & $30 \mathrm{~s}$ & $26(38.3 \%)$ \\
\cline { 2 - 3 } & $40 \mathrm{~s}$ & $10(14.7 \%)$ \\
\cline { 2 - 3 } & over 50s & $3(4.4 \%)$ \\
\hline
\end{tabular}

The survey was conducted online and offline for two weeks from 3rd July 2020, and the questionnaire contained total 39 questions including 4 questions on demographic characteristics, 20 questions on the effect of the affordance design factor on user experience and 15 questions on the degree of effect on the brand experience. Each question had 5-point scale, and the composition of the questionnaire is shown in Table 6.

Table 6: Survey composition

\begin{tabular}{|c|c|c|c|}
\hline \multicolumn{3}{|c|}{ questionnaire question } & number of question \\
\hline \multicolumn{3}{|c|}{ demographic characteristics } & 4 \\
\hline \multirow{5}{*}{$\begin{array}{l}\text { affordance design } \\
\text { elements }\end{array}$} & \multirow{5}{*}{ user experience factor } & ease of learning & 4 \\
\hline & & efficiency & 4 \\
\hline & & memory remains & 4 \\
\hline & & error & 4 \\
\hline & & satisfaction & 4 \\
\hline \multirow{3}{*}{ usability factor } & \multirow{3}{*}{$\begin{array}{l}\text { brand experience } \\
\text { factor }\end{array}$} & value & 5 \\
\hline & & reliability & 5 \\
\hline & & loyalty & 5 \\
\hline
\end{tabular}

\subsubsection{BX evaluation analysis result}

Frequency analysis and technical statistics analysis by factor of affordance design on usability according to user experience were conducted, and the results were derived as shown in Table 7. In the case of user experience's ease of learning, the learned form was 4.41, and the metaphor utilization was 4.32, which were higher than the contextual continuity and use relationship. In the case of UI utilization efficiency, as for ease of learning, the learned form ranked 4.29, and metaphor utilization ranked as high as 4.05. In the case of the memory possibility that the user's memory remains for a long time, the learning form showed the highest preference frequency of 4.52 , the metaphor utilization scored a relatively high score of 4.05 , the contextual continuity was 3.26 , and the use relationship was relatively low affordance design factor with 3.11.

Table 7: User experience impact of affordance design

\begin{tabular}{|c|c|c|c|c|}
\hline division & $\begin{array}{c}\text { metaphor } \\
\text { utilization }\end{array}$ & learned form & $\begin{array}{c}\text { contextual } \\
\text { continuity }\end{array}$ & use relationship \\
\hline ease of learning & 4.32 & 4.41 & 3.46 & 3.68 \\
\hline efficiency & 4.05 & 4.29 & 3.85 & 3.76 \\
\hline memory remains & 4.03 & 4.52 & 3.26 & 3.11 \\
\hline error prevention & 3.84 & 3.22 & 4.03 & 4.15 \\
\hline satisfaction & 4.16 & 3.52 & 3.86 & 3.97 \\
\hline
\end{tabular}

Table 8 shows the priority of the effect of the factors of affordance design on the user experience. In the case of the learned form, the user induces the UI behavior of the flight reservation system through the form of a push button by recognition that has already been learned, the direction using arrows, etc., so that learning ease and efficiency are high, and the possibility of memory due to the form already learned appeared high. In addition, icons and images using the metaphor technique were analyzed as an affordance design factor that is useful for ease of learning, efficiency, and memorization as the dictating meaning is delivered faster than text. In the case of error prevention, the relationship between each other on a single screen, i.e. there is an empty part in the text input box for boarding the aircraft, and the induction of actions such as deactivation of the button to the next step has been shown to minimize errors in the journey to achieve the user's purpose. It was also analyzed that inducing actions continuously, such as selecting the departure date and then selecting the arrival date after selecting the flight departure date or revealing the boarding flight information after entering information for flight reservation, prevents errors. However, in the case of satisfaction, the use of metaphor satisfies both aesthetics and usability, so it was derived as the design factor with the highest impact.

Table 8: Priority of the impact of affordance design elements on the user experience

\begin{tabular}{|c|c|}
\hline user experience & impact of affordance design on user experience \\
\hline ease of learning & learned form $>$ metaphor utilization $>$ use relationship $>$ contextual continuity \\
\hline efficiency & learned form $>$ metaphor utilization $>$ contextual continuity $>$ use relationship \\
\hline memory remains & learned form $>$ metaphor utilization $>$ contextual continuity $>$ use relationship \\
\hline error prevention & use relationship $>$ contextual continuity $>$ metaphor utilization $>$ learned form \\
\hline satisfaction & metaphor utilization $>$ use relationship $>$ contextual continuity $>$ learned form \\
\hline
\end{tabular}


Table 9 shows the effect of usability factors such as ease of learning, efficiency, memory possibility, error prevention, and satisfaction on value, reliability, and loyalty, which are measurement factors of brand experience. In the case of Brand value, satisfaction was 4.36 the highest, efficiency was 4.06, and learning ease was 3.35 , the lowest. In the case of reliability, error prevention was 4.87 the highest, and the memory probability factor was also high at 4.20, but the relatively low impact was analyzed as learning ease at 3.25, and efficiency at 3.28. In the case of brand loyalty, satisfaction was analyzed to show the highest impact at 4.85 , and learning ease was relatively high at 4.13 , followed by error prevention 3.98 , efficacy 3.87 , and memory possibility 3.69 , which was higher than other brand experience factors.

Table 9: Brand experience impact of user experience

\begin{tabular}{|c|c|c|c|c|c|}
\hline division & $\begin{array}{c}\text { ease of } \\
\text { learning }\end{array}$ & efficiency & $\begin{array}{c}\text { memory } \\
\text { remains }\end{array}$ & $\begin{array}{c}\text { error } \\
\text { prevention }\end{array}$ & satisfaction \\
\hline value & 3.35 & 4.06 & 3.66 & 3.95 & 4.36 \\
\hline reliability & 3.25 & 3.28 & 4.20 & 4.87 & 3.86 \\
\hline loyalty & 4.13 & 3.87 & 3.69 & 3.98 & 4.85 \\
\hline
\end{tabular}

The result of measuring the effect of the usability factor of user experience on the brand experience is shown in Table 10 below. As for the impact on the brand value which is a measurement factor of the brand experience, the satisfaction that users feel while using the flight reservation application was found to be the greatest.

As the flight reservation process takes place while the customer directly uses the application, overall satisfaction with the application was analyzed to be the biggest factor that determines the brand's value. It was analyzed that the efficiency of application use felt by users through the application UI also forms a high brand value.

In terms of brand reliability, the UI environment where user errors do not occur as much as possible during the process of using the flight reservation application showed the highest impact. In addition, it was analyzed that the UI design remains well in the memory and the memory possibility that the user's actions are naturally connected when visiting again shows a high degree of impact.

Brand loyalty was the user's idea to continue using the brand, indicating the highest impact on application use satisfaction, followed by ease of learning and error prevention.

However, in the case of loyalty, most factors of usability show a relatively high level of influence, so it was analyzed that the brand loyalty increases if most factors of usability are satisfied.

Table 8: Priority of the impact of usability factors on brand experience

\begin{tabular}{|c|c|}
\hline brand experience & impact of usability factor on brand experience \\
\hline value & satisfaction $>$ efficiency $>$ error prevention $>$ memory remains $>$ ease of learning \\
\hline reliability & error prevention $>$ memory remains $>$ satisfaction $>$ efficiency $>$ ease of learning \\
\hline loyalty & satisfaction $>$ ease of learning $>$ error prevention $>$ efficiency $>$ memory remains \\
\hline
\end{tabular}

\section{Conclusion}

This study attempted to derive a design plan by measuring the impact of the usability of the UI design of an affordance-based flight reservation application on the airline brand experience. Therefore, this study analyzed the impact of the affordance based flight reservation application UI design on the improvement of the airline's brand value, reliability and loyalty.

The result of affordance design BX analysis of this study is meaningful in that it can be used as basic data for planning, development, and design to provide more improved and convenient contents to users using flight reservation applications. In addition, the research results derived through this study can be used as a way to apply the affordance of various application UI designs other than flight reservation applications and create brand value by improving the UI design of applications that are amplifying in the mobile first era, and is expected to have a positive impact on the IT-based service industry.

\section{References}

1. Korea Culture and Tourism Institute. (2019) 2018 Korea National Tourism Survey. Korea: Ministry of Culture, Sports and Tourism, 8-19.

2. K. B. Yoon and S. H. Song. (2013) Study on the utilization of Travel site Matjip (Reputable Restaurant) search application(APP) using Smart Phone. Journal of Digital Convergence, 11(10), 437-443.

3. S. H. Kim. (2014) A Study on Affordance Design to Induce User`s Cognitive Experiences -Focus on Smart UI Usability. Journal of the Korean Society of Design Culture, 20(4), 87-96.

4. Nielsen Norman Group. (2012) Usability 101: Introduction to Usability. Retrieved from: https://www.nngroup.com/articles/usability-101-introduction-to-usability/ (website)

5. J. Brakus., Bernd H. Schmitt and Lia Zarantonello. (2009) Brand Experience : What Is It? How Is It Measured? Does It Affect Loyalty?. , 73(May). 
6. Dong-A Business Review. (2014) Gray Holland's "cycle of experience". Retrieved from: https://dbr.donga.com/graphic/view/gdbr_no/3824 (website)

7. H. S. Cho. (2017) A Study on Preference for Graphic Basic Elements of the UI/UX Design of Game Application. Journal of Digital Convergence, 15(12), 573-579.

8. Y. J. Lee. (2019) Chatbot UX in a Mobile Environment. Journal of Digital Convergence, 17(11), 517522.

9. C. S. Kim. (2014) A Conceptual Study on Brand Experience(BX). Journal of Brand Design Association of Korea, 12(3), 121-130.

10. S. M. Kim. (2017) A Study on the Effects of BX Design Experience on the Value Perception, Reliability, and Loyalty of Brand. Journal of Communication Design, 61, 99-110. 